\title{
Avaliação de Dados Hiperespectrais como Preditores para Biomassa de Campos Nativos
}

\author{
Evaluation of Hyperspectral Data as Predictors for Natural Grassland \\ Biomass
}

Marildo Guerini Filho ${ }^{1}$
Tatiana Mora Kuplich ${ }^{2}$

Recebido em agosto de 2018.

Aprovado em agosto de 2019.

\begin{abstract}
RESUMO
O Bioma Pampa representa 63\% do território do Rio Grande do Sul - Brasil. Em função da contínua incorporação de monoculturas de espécies exóticas, cultivos agrícolas e a práticas por vezes inadequadas de produção pecuária, os campos nativos estão rapidamente sendo degradados, fragmentados e descaracterizados. Buscando colaborar no desenvolvimento de novas estratégias de manejo e monitoramento adequado dos campos nativos, com vistas à minimização de esforços para coletas de campo, esta pesquisa objetivou caracterizar e quantificar a relação entre dados hiperespectrais coletados por espectrorradiômetro como preditores de biomassa campestre em duas alternativas de manejo pastoril com o auxílio de dados de campo. A área de estudo são parcelas pastoreadas por bovinos em duas alternativas de manejo (375 e 750 graus dia - GD) de onde foram adquiridas curvas espectrais de reflectância com espectrorradiômetro ao longo do intervalo de 350-2500 nm de comprimento de onda (CO). Foram calculados 10 índices de vegetação e utilizados juntamente com 11 intervalos de $\mathrm{CO}$ nas análises de regressão. Os dados espectrais simularam os $\mathrm{CO}$ utilizados nas bandas do sensor MSI do satélite Sentinel 2, disponíveis gratuitamente desde 2015. Os resultados mostraram excelentes correlações entre as variáveis e verificouse que nas regiões espectrais do Azul, Red edge e os índices NDLI e DMCI diferiram estatisticamente entre as alternativas de manejo. As regiões espectrais do Azul, NIR e SWIR foram significativamente maiores no tratamento $750 \mathrm{GD}$. O modelo mais preciso para estimar biomassa envolveu os índices EVI e CAI com $\mathrm{R}^{2}$ ajustado $=0.72$ e RMSE $=0.10$.
\end{abstract}

PALAVRAS-CHAVE: Campos nativos. Biomassa vegetal. Dados remotos. Espectrorradiômetria. Geociência.

\footnotetext{
${ }^{1}$ Universidade Federal do Rio Grande do Sul; Centro Estadual de Pesquisas em Sensoriamento Remoto e Meteorologia; guerinimarildo@gmail.com

2 Instituto Nacional de Pesquisas Espaciais; Universidade Federal do Rio Grande do Sul; Centro Estadual de Pesquisas em Sensoriamento Remoto e Meteorologia; tkuplich@gmail.com
} 


\begin{abstract}
The Pampa Biome represents approximately $63 \%$ of the territory in the State of Rio Grande do Sul - Brazil. Due to the continuous incorporation of monocultures of exotic species, agricultural crops and sometimes inadequate practices of livestock production, native fields are rapidly being degraded, fragmented and uncharacterized. Seeking to collaborate in developing new management strategies and appropriate monitoring of natural grassland, with a view to minimizing efforts for field collection, this study aimed to characterize and quantify the relationship between hyperspectral data collected by spectroradiometer as biomass predictors country in two methods pasture management with the aid of field data. The study area are plots of grazing cattle by two methods (treatments) management (day 375 to 750 degrees - DG) which were acquired spectral reflectance curves with spectroradiometer over the range $350-2500 \mathrm{~nm}$ wavelength. 10 vegetation indices were used and used along with $11 \mathrm{CO}$ intervals in the regression analyzes. Selected indices and ranges simulated wavelength used in the Sentinel 2 satellite MSI sensor bands, available free of charge since 2015. The results showed strong correlations between the variables and it was found that in the spectral regions of blue, red edge and NDLI and DMCI differed statistically among management methods. The spectral regions of Blue, NIR and SWIR were significantly higher in 750 GD treatment. The most accurate model to biomass estimation involved the EVI and CAI indices with adjusted $\mathrm{r}^{2}=0.72$ and $\mathrm{RMSE}=0.10$.
\end{abstract}

KEYWORDS: Natural grassland. Plant biomass. Remote data. Spectroradiometry. Geoscience.

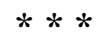

\title{
Introdução
}

Os campos nativos do Bioma Pampa representam aproximadamente 63\% do território do Rio Grande do Sul - Brasil, porém, estima-se que restam apenas 36\% de sua área original (CORDEIRO e HASENACK, 2009). São amplamente conhecidos pela alta biodiversidade de espécies de fauna e flora. No entanto, práticas por vezes inadequadas de produção pecuária, a introdução de espécies arbóreas exóticas e a conversão de campos para agricultura vêm degradando e fragmentando o Bioma em um ritmo bastante acelerado (OVERBECK et al., 2009).

O Pampa é responsável por importantes serviços ambientais e tem função reguladora nos ciclos de matéria (vegetação, fauna, flora...) e energia geradas pelos ecossistemas naturais, mantendo o ambiente equilibrado 
(BOLFE, BATISTELLA e FERREIRA, 2012). A forma que os campos são manejados e as condições ambientais da região influenciam diretamente na sua capacidade de regeneração, alterando sua composição vegetal e também a quantidade de biomassa. A biomassa dos campos nativos apresenta-se como uma importante variável para controle e monitoramento da dinâmica dos campos e seus efeitos em relação biodiversidade de fauna e flora (NUMATA et al., 2007).

Os estudos que utilizam técnicas de sensoriamento remoto (SR) para monitoramento, controle e avaliação de variáveis biofísicas da vegetação campestre são escassos quando comparados a outros tipos de vegetação (HILL, 2013; ULLAH et al., 2012; YANG et al., 2015). Considerando as limitações de imagens de satélites de média resolução espectral (e.g., Landsat, Terra e Acqua), as relações com a biomassa e os dados de SR muitas vezes são insatisfatórias. Na vegetação campestre, onde a matéria senescente é parte importante do dossel, estas relações podem se apresentar ainda mais fracas, já que a maior parte dos índices de vegetação, usados frequentemente nas estimativas de biomassa, mede o vigor da vegetação verde.

Os dados de espectrorradiômetria possibilitam adquirir espectros instantaneamente, permitindo uma vasta gama de combinações e relações entre os comprimentos de onda amostrados e, principalmente, fornecem informações em faixas do espectro não disponibilizadas por imagens orbitais de média resolução espectral. Em especial, as faixas específicas para análises de biomassa seca ou senescente, geralmente no infravermelho médio (ou de ondas curtas), estão disponíveis em determinados espectrorradiômetros.

Neste contexto, buscando colaborar no desenvolvimento de novas estratégias de manejo e monitoramento adequado dos campos nativos, com vistas à minimização de esforços para coletas de campo, esta pesquisa objetivou caracterizar e quantificar a relação entre dados hiperespectrais como preditores de biomassa campestre em dois métodos de manejo pastoril com o auxílio de dados de campo. 


\section{Materiais e métodos}

Em linhas gerais, na Figura 1 é apresentado o fluxograma metodológico para realização do estudo.

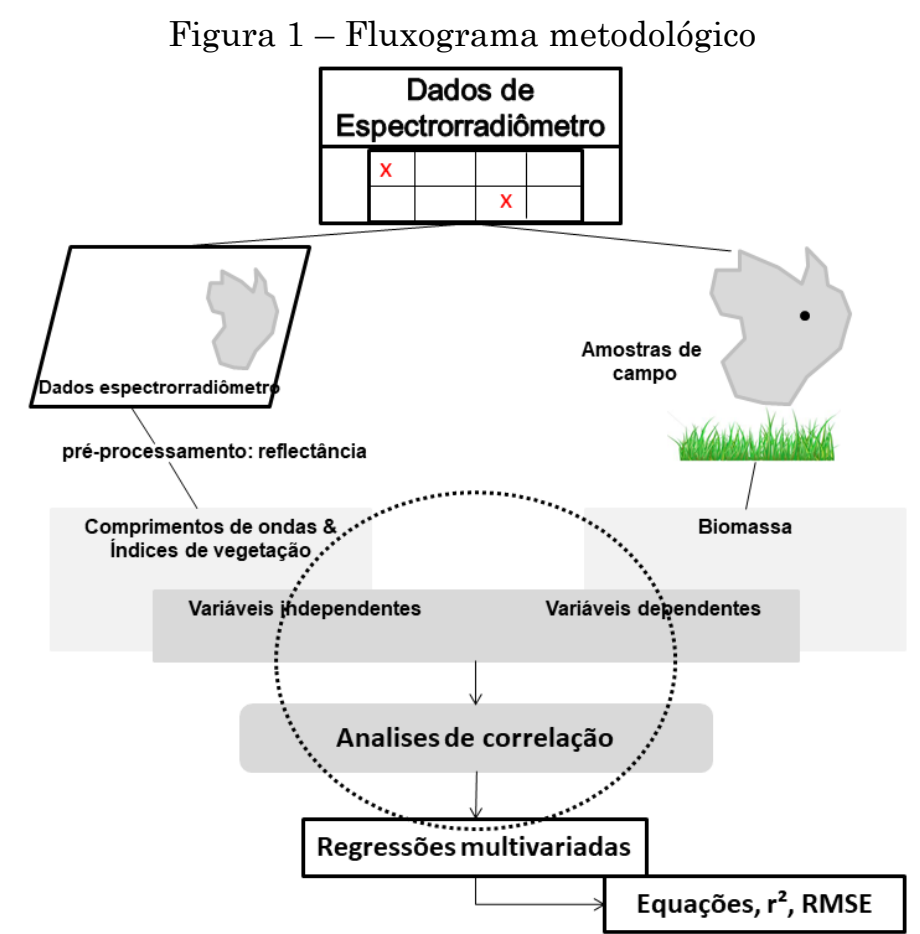

Fonte: Elaborada pelos autores.

1.1 Área de estudo

O estudo foi realizado em área de campo nativo pertencente à Universidade Federal de Santa Maria (UFSM) possuindo 23 hectares, localizada na região fisiográfica Depressão Central do Rio Grande do Sul com

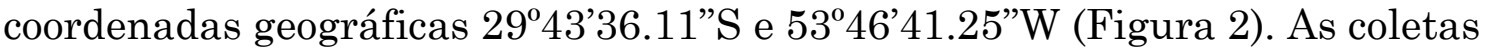
de campo foram realizadas em novembro de 2017. A área apresenta clima subtropical úmido (Cfa) segundo a classificação de Köppen e está a 95 m de altitude. Santa Maria tem pluviosidade média anual de $1688 \mathrm{~mm}$ e média de temperatura mínima de $19.4{ }^{\circ} \mathrm{C}$ e máxima de $30.4^{\circ} \mathrm{C}$ mensal (STRECK, 2008). 
A área é dividida em duas formas de manejo pastoril, chamados tratamentos, baseadas na soma térmica acumulada para o crescimento médio foliar de dois grupos funcionais de gramíneas classificados conforme o estudo proposto por Cruz et al. (2010). Estes tratamentos, com intervalos de 375 e 750 graus dia (GD), consideram o tempo necessário, em dias, para a temperatura atmosférica atingir o total de 375 e 750 graus, quando o gado é introduzido para pastejo.

A área é dividida em unidades experimentais (piquetes) de aproximadamente 0,5 ha cada. Para a pesquisa, selecionou-se previamente 5 piquetes em cada tratamento.

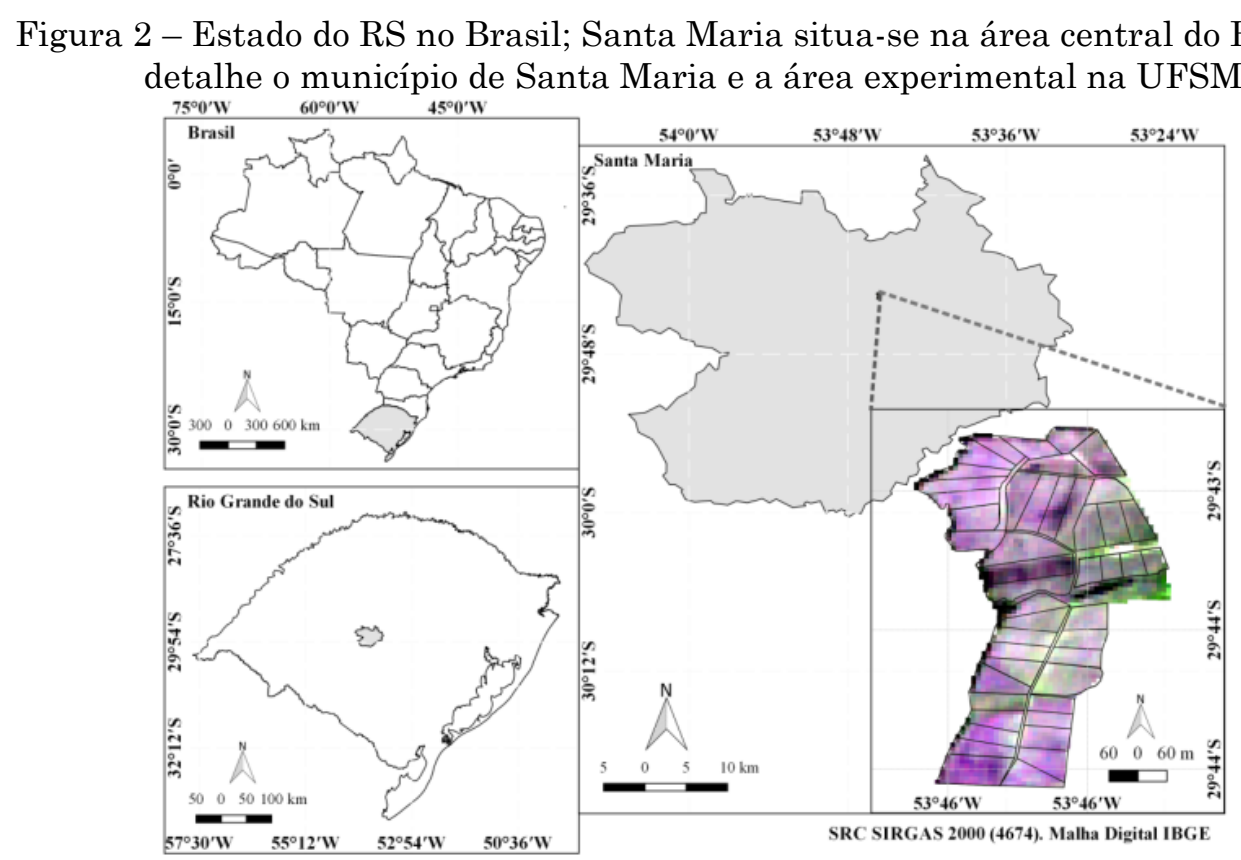

Fonte: Elaborada pelos autores.

\subsection{Dados do espectrorradiômetro}

Os dados hiperespectrais foram coletados com espectrorradiômetro portátil ASD FieldSpec ${ }^{\circledR} 3$ que registra dados na faixa de $350-2500 \mathrm{~nm}$ de comprimento de onda. Foram selecionados dez piquetes entre os dois tratamentos de manejo pastoril. As medições para cada piquete foram feitas em tempo com alta luminosidade, sem presença de nuvens no céu. Todos as 
medidas foram feitas com ângulo de visada de $90^{\circ}$ na direção do norte e a cerca de $1 \mathrm{~m}$ acima do dossel da vegetação, gerando assim um campo de visada de aproximadamente 0.5 x $0.5 \mathrm{~m}$. Um painel de referência branco Spectralon foi utilizado para medição e calibração antes das medidas da reflectância da vegetação. A calibração prévia permite a conversão das medidas de radiação espectral em reflectância. Foram realizadas dez leituras para cada unidades amostral e gerada a média para os espectros finais. As medições foram tomadas no período das 11:00 às 15:00 horas.

Para melhor compreensão das variações dos comprimentos de onda em cada piquete e tratamento, o espectro foi previamente dividido em intervalos que estão apresentados na Tabela 1. Os intervalos foram definidos com base nas bandas das imagens MSI (Multispectral Imager) dos satélites Sentinel 2.

Tabela 1 - Divisão do espectro em intervalos

\begin{tabular}{c|c|c}
\hline \multirow{2}{*}{ ID* } & \multicolumn{2}{|c}{ Intervalo (nm)** } \\
\cline { 2 - 3 } & Inicio & Fim \\
\hline Ivis & 400 & 700 \\
\hline INIR & 700 & 1300 \\
\hline ISWIR 1 & 1301 & 1900 \\
\hline ISWIR 2 & 1901 & 2500 \\
\hline Ib & 447 & 546 \\
\hline Ig & 538 & 582 \\
\hline Ir & 646 & 669 \\
\hline Ire & 695 & 715 \\
\hline Ire1 & 731 & 749 \\
\hline Iir & 762 & 907 \\
\hline Is & 2000 & 2200 \\
\hline
\end{tabular}

Fonte: Elaborada pelos autores.

*Ivis: intervalo na faixa do visivel; INIR: intervalo faixa do infravermelho próximo; ISWIR: intervalo faixa do infravermelho de ondas curtas; Ib: intervalo faixa do azul; Ig: intervalo faixa do verde; Ir: intervalo faixa do vermelho: Ire: intervalo faixa do rededge; Iir: intervalo faixa do infravermelho; intervalo na faixa da senescência da vegetação. ** Os intervalos foram baseados na largura das bandas do satélite Sentinel-2. 


\section{3 Índices de vegetação}

Esta pesquisa explorou $10 \mathrm{IVs}$ (Tabela 2), alguns amplamente conhecidos por suas correlações com a biomassa verde (GONZÁLEZ-DUGO e MATEOS, 2008; HMIMINA et al., 2013; XU et al., 2014) e outros por avaliar a biomassa senescente (NAGLER et al., 2003; WANG et al., 2011b).

Tabela 2 - Índices de vegetação utilizados

\begin{tabular}{|c|c|c|c|}
\hline Índice & Nome & Citação & Fórmula* \\
\hline EVI & $\begin{array}{l}\text { Enhanced vegetation } \\
\text { index }\end{array}$ & Huete et al. (1997) & $\begin{array}{c}\mathrm{B}=2.5(\text { lre }-\operatorname{lr}) /(\text { lre }+6 \times \operatorname{lr}-7.5 \\
\times \mathrm{lb}+1)\end{array}$ \\
\hline EVI8 & $\begin{array}{c}\text { Enhanced vegetation } \\
\text { index }\end{array}$ & Huete et al. (1997) & $\begin{array}{c}\mathrm{B}=2.5(\operatorname{lir}-\operatorname{lr}) /(\operatorname{lir}+6 \times \operatorname{lr}-7.5 \times \\
\mathrm{lb}+1)\end{array}$ \\
\hline NDREI & $\begin{array}{l}\text { Normalized difference } \\
\text { red edge index }\end{array}$ & $\begin{array}{c}\text { Gitelson e Merzlyak } \\
(1994) \\
\end{array}$ & $\mathrm{B}=(\mathrm{lre}-\mathrm{lr}) /(\mathrm{lre}+\mathrm{lr})$ \\
\hline NDVI & $\begin{array}{l}\text { Normalized difference } \\
\text { vegetation index }\end{array}$ & Tucker (1979) & $\mathrm{B}=(\operatorname{lir}-\operatorname{lr}) /(\mathrm{lir}+\mathrm{l} r)$ \\
\hline PSRI & $\begin{array}{l}\text { Plant senescence } \\
\text { reflectance index }\end{array}$ & $\begin{array}{l}\text { Merzlyak et al. } \\
\text { (1999) }\end{array}$ & $B=\rho 681-\rho 498 / \rho 752$ \\
\hline NDLI & $\begin{array}{c}\text { Normalized difference } \\
\text { lignin index } \\
\end{array}$ & $\begin{array}{l}\text { Serrano, Penuelas e } \\
\text { Ustin (2002) } \\
\end{array}$ & $\begin{array}{c}\mathrm{B}=[\log (1 / \rho 1754)-\log (1 / \rho 1680)] / \\
{[\log (1 / \rho 1754)+\log (1 / \rho 1680)]}\end{array}$ \\
\hline $\begin{array}{l}\text { MREND } \\
\text { VI }\end{array}$ & Modified red edge NDVI & $\begin{array}{c}\text { Sims e Gamon } \\
(2002)\end{array}$ & $\begin{array}{c}\mathrm{B}=(\rho 750-\rho 705) /(\rho 750+\rho 705- \\
\left.2^{*} \rho 445\right)\end{array}$ \\
\hline NDMI & $\begin{array}{l}\text { Normalized dry matter } \\
\text { index }\end{array}$ & $\begin{array}{l}\text { Wang et al. (2011a, } \\
2011 \mathrm{~b})\end{array}$ & $\begin{array}{c}\mathrm{B}=(\rho 1649-\rho 1722) /(\rho 1649+ \\
\rho 1722)\end{array}$ \\
\hline CAI & $\begin{array}{l}\text { Cellulose absorption } \\
\text { index }\end{array}$ & Nagler et al. (2003) & $B=0.5(\rho 2031+\rho 2211)-\rho 2101$ \\
\hline DMCI & Dry matter content index & $\begin{array}{l}\text { Romero, Aguado e } \\
\text { Yebra (2012) }\end{array}$ & $\begin{array}{c}B=(\rho 2305-\rho 1495) /(\rho 2305+ \\
\rho 1495)\end{array}$ \\
\hline
\end{tabular}

\section{Fonte: Elaborada pelos autores.}

* $\rho$ é reflectância e o subíndice é comprimento de onda (nm). lre é banda na faixa do rededge. lr é a banda do vermelho. lb representa a banda do azul e lir utiliza a banda do infravermelho.

\subsection{Amostragem de biomassa campestre}

Os dados de campo foram coletados no mês de novembro de 2017 com a colaboração da equipe técnica do Laboratório de Ecologia de Pastagens Naturais (LEPAN) do Departamento do Zootecnia da Universidade Federal de Santa Maria (Figura 3). 
Figura 3 - a) momento da coleta das unidades amostrais de biomassa e dados do espectrorradiômetro; b) fisionomia característica do tratamento 375 GD; c) fisionomia característica do tratamento $750 \mathrm{GD}$

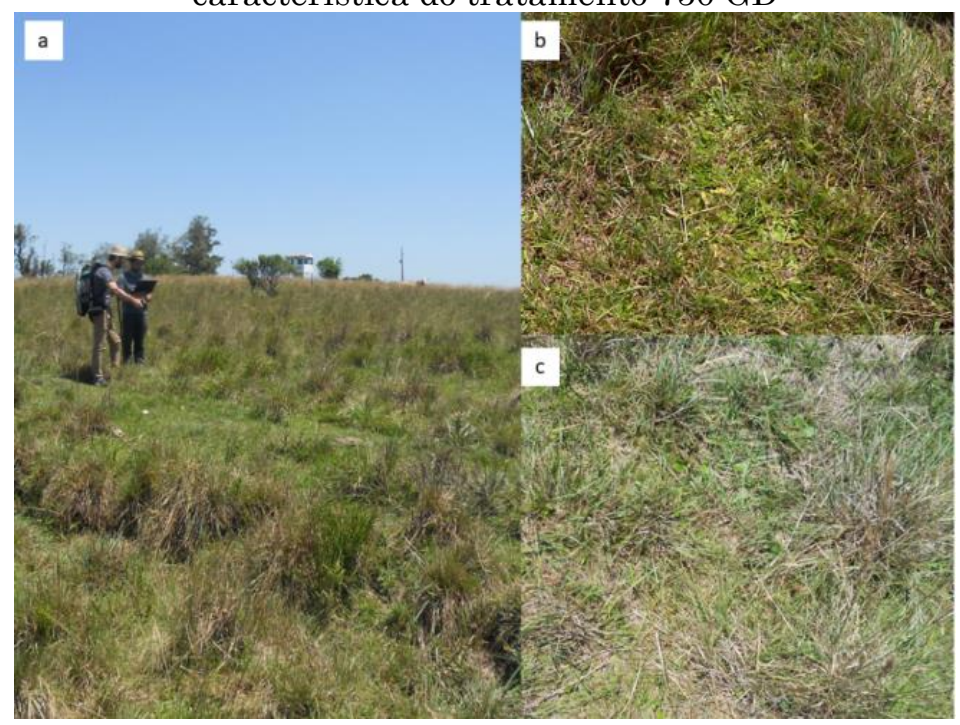

Fonte: Elaborada pelos autores.

A biomassa total das unidades amostrais $(50 \times 50 \mathrm{~cm})$ foram determinadas através da técnica de estimativa visual por comparação de padrões, calibrada com duas amostragens (Haydock e Shaw, 1975), com 10 estimativas visuais em cada piquete. Já para a estimativa do percentual de biomassa verde e senescente foi realizada a avaliação visual por meio de fotos especificas de cada unidade amostral conforme descrito pelo Instituto Nacional de Investigação Agropecuária do Uruguai. Foram anotadas as medidas de altura do dossel, com o auxílio de régua graduada e foi realizada a coleta dos espectros utilizando o equipamento FieldSpec® 3. Em uma análise preliminar, visualmente, foi possível verificar maior presença de touceiras e biomassa senescente nos piquetes do tratamento 750 GD.

\subsection{Análises estatísticas}

Para o processamento dos dados utilizou-se os softwares estatísticos RStudio 1.0.153 e SPSS 22.0. A normalidade dos dados foi testada através do teste de Kolmogorov-Smirnov. Foram calculadas estatísticas descritivas (médias e desvios-padrão, frequências, percentuais) e bivariadas (Mann- 
Whitney, ANOVA, testes $\mathrm{t}$ de Student para amostras independentes, Kruskal-Wallis, Spearman, Pearson e regressão linear múltipla).

A relação entre as variáveis espectrais (na forma de índices) e a biomassa campestre foi inferida por meio de modelos de regressão linear múltipla de primeira ordem, dado pela expressão:

$$
B=\theta_{0}+\theta_{1} X+\theta_{2} Y \ldots+\theta_{n} N+\epsilon
$$

em que, $\theta_{0}, \theta_{1}, \theta_{2}$ e $\theta$ n são coeficientes desconhecidos, $\mathrm{X}, \mathrm{Y}$ e $\mathrm{N}$ são os dados espectrais e $\epsilon$ é o erro aleatório. Em todas as expressões aplicou-se métodos para verificação dos pressupostos p-valor, multicolinearidade, homogeneidade, independência dos erros e normalidade dos resíduos. O desempenho dos modelos de regressão foi avaliado aplicando os testes de $\mathrm{t}$ de Student e Mann Whitney. A acurácia dos modelos foi avaliada mediante o

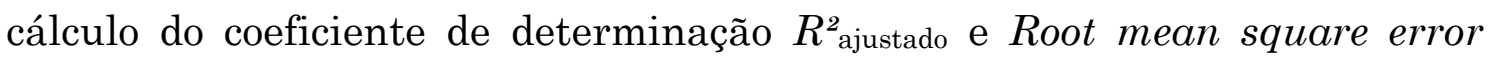
(RMSE).

\section{Resultados}

Foram incluídos na pesquisa 96 pares de dados de biomassa e espectros coletados com espectrorradiômetro, sendo 50 pares $(52,1 \%)$ referentes a 5 piquetes no tratamento de 375 GD e 46 pontos (47,9\%) referentes a 5 piquetes no tratamento de 750 GD. Na Figura 4 são apresentados os espectros médios (10 leituras) para cada piquete e tratamento. Os piquetes 1 a 5 foram medidos no tratamento 375 e os piquetes 6 ao 10 no tratamento 750 . Em função de ruídos nos extremos do espectro e nos picos de absorção da água, os intervalos entre 350-400, 1360-1400, 1800-1950 e 2400-2500 nm foram removidos das análises. Analisando os valores médios de reflectância dos intervalos do espectrorradiômetro descritos na Tabela 2 observou-se que o INIR, ISWIR1, ISWIR2, Ib e Is foram significativamente maiores no tratamento $750 \mathrm{GD}$ $(p<0.05)$, os demais intervalos não diferiram significativamente. Dentro de cada tratamento também foram analisadas as diferenças entre os piquetes e 
verificou-se alta variabilidade com diferenças significativas na refletância entre todos nos dois tratamentos $(\mathrm{p}<0.05)$ Tabela 3.

Figura 4 - Curvas espectrais médias dos, a) piquetes e b) tratamento
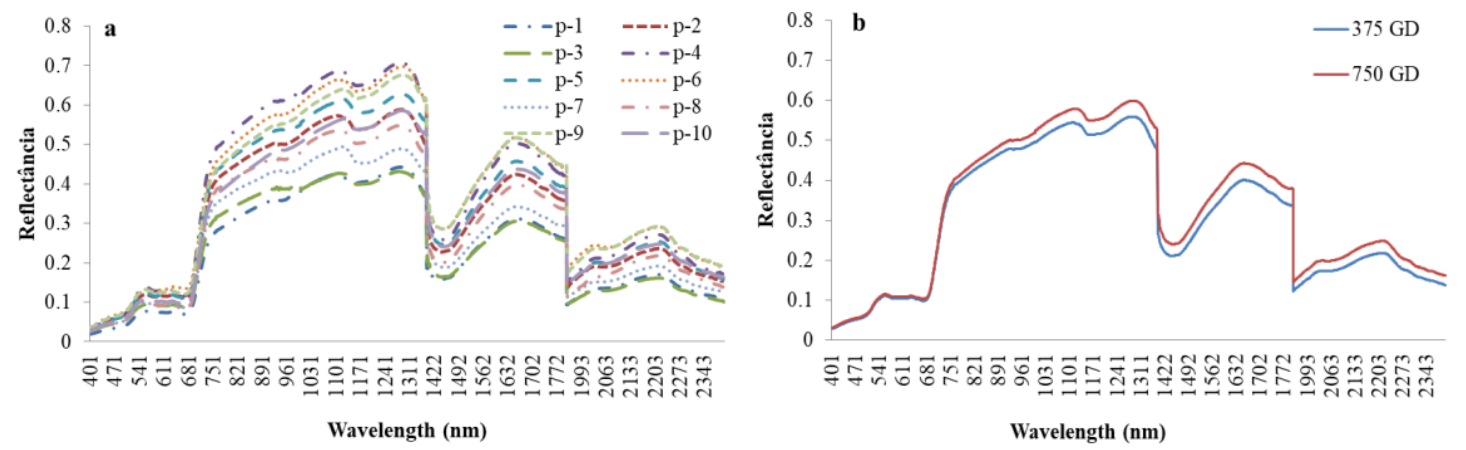

Fonte: Elaborada pelos autores.

Tabela 3 - Comparação entre os piquetes

\begin{tabular}{|c|c|c|c|c|c|c|c|c|}
\hline \multirow{2}{*}{ Variável* } & \multirow{2}{*}{ Piquete } & \multicolumn{2}{|c|}{375 GD } & \multirow{2}{*}{$p^{* * *}$} & \multicolumn{2}{|c|}{750 GD } & \multirow{2}{*}{$p^{* * * * *}$} & \multirow{2}{*}{$p^{* * * * * *}$} \\
\hline & & Média & DP & & Média & DP & & \\
\hline \multirow{5}{*}{ Ivis } & 1 & 0.061 & 0.024 & \multirow{5}{*}{$<0.001$} & 0.104 & 0.038 & \multirow{5}{*}{$<0.001$} & \multirow{5}{*}{0.177} \\
\hline & 2 & 0.089 & 0.037 & & 0.075 & 0.028 & & \\
\hline & 3 & 0.073 & 0.028 & & 0.068 & 0.027 & & \\
\hline & 4 & 0.112 & 0.045 & & 0.100 & 0.035 & & \\
\hline & 5 & 0.098 & 0.038 & & 0.085 & 0.032 & & \\
\hline \multirow{5}{*}{ INIR } & 1 & 0.372 & 0.089 & \multirow{5}{*}{$<0.001$} & 0.579 & 0.117 & \multirow{5}{*}{$<0.001$} & \multirow{5}{*}{$<0.001$} \\
\hline & 2 & 0.532 & 0.104 & & 0.422 & 0.088 & & \\
\hline & 3 & 0.386 & 0.074 & & 0.446 & 0.099 & & \\
\hline & 4 & 0.625 & 0.129 & & 0.561 & 0.110 & & \\
\hline & 5 & 0.553 & 0.109 & & 0.504 & 0.096 & & \\
\hline \multirow{5}{*}{ ISWIR1 } & 1 & 0.280 & 0.083 & \multirow{5}{*}{$<0.001$} & 0.446 & 0.120 & \multirow{5}{*}{$<0.001$} & \multirow{5}{*}{$<0.001$} \\
\hline & 2 & 0.370 & 0.134 & & 0.308 & 0.090 & & \\
\hline & 3 & 0.270 & 0.079 & & 0.308 & 0.106 & & \\
\hline & 4 & 0.472 & 0.137 & & 0.450 & 0.119 & & \\
\hline & 5 & 0.425 & 0.124 & & 0.396 & 0.098 & & \\
\hline \multirow{5}{*}{ ISWIR2 } & 1 & 0.145 & 0.029 & \multirow{5}{*}{$<0.001$} & 0.235 & 0.044 & \multirow{5}{*}{$<0.001$} & \multirow{5}{*}{$<0.001$} \\
\hline & 2 & 0.189 & 0.073 & & 0.162 & 0.042 & & \\
\hline & 3 & 0.132 & 0.031 & & 0.151 & 0.044 & & \\
\hline & 4 & 0.246 & 0.059 & & 0.236 & 0.049 & & \\
\hline & 5 & 0.220 & 0.053 & & 0.213 & 0.035 & & \\
\hline \multirow{5}{*}{$\mathrm{Ib}$} & 1 & 0.048 & 0.016 & \multirow{5}{*}{$<0.001$} & 0.083 & 0.023 & \multirow{5}{*}{$<0.001$} & \multirow{5}{*}{0.030} \\
\hline & 2 & 0.074 & 0.026 & & 0.062 & 0.019 & & \\
\hline & 3 & 0.059 & 0.019 & & 0.057 & 0.020 & & \\
\hline & 4 & 0.089 & 0.030 & & 0.082 & 0.023 & & \\
\hline & 5 & 0.080 & 0.026 & & 0.068 & 0.021 & & \\
\hline \multirow{5}{*}{$\mathrm{Ig}$} & 1 & 0.078 & 0.012 & \multirow{5}{*}{$<0.001$} & 0.129 & 0.015 & & \\
\hline & 2 & 0.116 & 0.021 & & 0.098 & 0.012 & & \\
\hline & 3 & 0.093 & 0.012 & & 0.093 & 0.015 & $<0.001$ & 0.492 \\
\hline & 4 & 0.148 & 0.021 & & 0.129 & 0.013 & & \\
\hline & 5 & 0.129 & 0.019 & & 0.112 & 0.009 & & \\
\hline
\end{tabular}




\begin{tabular}{|c|c|c|c|c|c|c|c|c|}
\hline \multirow{5}{*}{$\mathrm{Ir}$} & 1 & 0.077 & 0.012 & \multirow{5}{*}{$<0.001$} & 0.132 & 0.014 & \multirow{5}{*}{$<0.001$} & \multirow{5}{*}{0.434} \\
\hline & 2 & 0.108 & 0.030 & & 0.092 & 0.014 & & \\
\hline & 3 & 0.089 & 0.019 & & 0.079 & 0.016 & & \\
\hline & 4 & 0.137 & 0.019 & & 0.123 & 0.010 & & \\
\hline & 5 & 0.120 & 0.017 & & 0.103 & 0.016 & & \\
\hline \multirow{5}{*}{ Ire } & 1 & 0.145 & 0.034 & \multirow{5}{*}{$<0.001$} & 0.231 & 0.043 & \multirow{5}{*}{$<0.001$} & \multirow{5}{*}{0.992} \\
\hline & 2 & 0.196 & 0.047 & & 0.169 & 0.036 & & \\
\hline & 3 & 0.165 & 0.034 & & 0.161 & 0.041 & & \\
\hline & 4 & 0.260 & 0.053 & & 0.219 & 0.040 & & \\
\hline & 5 & 0.222 & 0.049 & & 0.200 & 0.038 & & \\
\hline \multirow{5}{*}{ Ire 1} & 1 & 0.260 & 0.046 & \multirow{5}{*}{$<0.001$} & 0.415 & 0.048 & \multirow{5}{*}{$<0.001$} & \multirow{5}{*}{0.941} \\
\hline & 2 & 0.396 & 0.053 & & 0.313 & 0.052 & & \\
\hline & 3 & 0.294 & 0.040 & & 0.333 & 0.053 & & \\
\hline & 4 & 0.460 & 0.068 & & 0.403 & 0.038 & & \\
\hline & 5 & 0.407 & 0.049 & & 0.356 & 0.030 & & \\
\hline \multirow{5}{*}{ Iir } & 1 & 0.326 & 0.059 & \multirow{5}{*}{$<0.001$} & 0.519 & 0.064 & \multirow{5}{*}{$<0.001$} & \multirow{5}{*}{0.330} \\
\hline & 2 & 0.495 & 0.068 & & 0.385 & 0.062 & & \\
\hline & 3 & 0.360 & 0.052 & & 0.413 & 0.065 & & \\
\hline & 4 & 0.563 & 0.084 & & 0.500 & 0.050 & & \\
\hline & 5 & 0.499 & 0.059 & & 0.442 & 0.045 & & \\
\hline \multirow{5}{*}{ Is } & 1 & 0.154 & 0.026 & \multirow{5}{*}{$<0.001$} & 0.250 & 0.039 & \multirow{5}{*}{$<0.001$} & \multirow{5}{*}{$<0.001$} \\
\hline & 2 & 0.200 & 0.073 & & 0.171 & 0.040 & & \\
\hline & 3 & 0.141 & 0.029 & & 0.160 & 0.042 & & \\
\hline & 4 & 0.262 & 0.055 & & 0.251 & 0.044 & & \\
\hline & 5 & 0.233 & 0.050 & & 0.225 & 0.029 & & \\
\hline
\end{tabular}

Fonte: Elaborada pelos autores.

*Ivis: intervalo na faixa do visivel; INIR: intervalo faixa do infravermelho próximo; ISWIR: intervalo faixa do infravermelho de ondas curtas; Ib: intervalo faixa do azul; Ig: intervalo faixa do verde; Ir: intervalo faixa do vermelho: Ire: intervalo faixa do rededge; Iir: intervalo faixa do infravermelho; intervalo na faixa da senescência da vegetação. ${ }^{* *}$ p referente a comparação entre tratamentos; ${ }^{* * *}$ p referente a comparação entre piquetes $375 \mathrm{GD} ; * * *$ p referente a comparação entre piquetes 750GD; Resultados descritos em média e desviopadrão (DP). p = nível de significância. Testes de ANOVA e Kruskal-Wallis para a comparação entre as variáveis, considerando significativo p<0,05 (IC 95\%).

Comparando os dados de reflectância (Ib, Ig, Ir, Ire, Iir e os índices de vegetação EVI, EVI8, NDREI, NDVI, PSRI, NDLI, MRENDVI, NDMI, DMCI, CAI) com os dados de campo (biomassa total - BT, biomassa verde total - BVT, porcentagem de biomassa verde - PBV, biomassa senescente total - BST, porcentagem de biomassa senescente - PBS) entre os tratamentos Tabela $4 \mathrm{e}$ Figura 5, verificou-se que no tratamento 375 GD a BT apresentou correlação inversa com DMCI e NDVI; a BST correlação inversa com o intervalo Iir, com os índices NDVI, DMCI, MRENDVI, NDREI, EVI8 e PBV e correlação direta com PSRI e PBS; já a BVT apresentou correlação inversa com os índices DMCI 
e PSRI e direta com EVI; os intervalos Ib, Ig, Ir, Ire e Iir apresentaram fortes correlações diretas entre eles e correlação inversa com NDLI; o PBV apontou correlação inversa com PSRI e CAI e direta com MRENDVI, NDVI e EVI8; a PBS apontou correlação inversa com MRENDVI, NDVI, NDREI, EVI8 e EVI.

No tratamento 750 GD observou-se correlação inversa da BT com os intervalos de reflectância nas faixas do Ib, Ig, Ir, Ire e Iir e com os índices PSRI e CAI, e correlação direta com NDLI; a BST mostrou correlação inversa com os intervalos Ib, Ig, Ir, Ire e Iir e com os índices EVI, EVI8 e NDMI e PBV; já a BVT apresentou forte correlação inversa com os intervalos Ib, Ig, Ir, Ire e Iir e com PSRI e CAI, e correlação direta com NDVI, NDLI, MRENDVI e PBV; os intervalos CAI, PSRI e os índices Ib, Ig, Ir, Ire e Iir apresentaram correlação inversa com PBV; e a PBS correlação Inversa com NDLI, NDVI, MRENDVI e NDREI.

As diferenças verificadas na região da banda do azul, no infravermelho e nos índices DMCI e NDLI podem naturalmente ser explicadas em função da quantidade de biomassa fotossinteticamente ativa entre os tratamentos. É possível verificar menor quantidade de biomassa senescente no tratamento 375 GD e maior quantidade de biomassa verde.

Tabela 4 - Diferença entre tratamentos

\begin{tabular}{c|c|c|c|c|c}
\hline \multirow{2}{*}{} & \multicolumn{2}{|c|}{ 375 GD } & \multicolumn{2}{c|}{$\mathbf{7 5 0}$ GD } & \multirow{2}{*}{ p } \\
\cline { 2 - 5 } & Média & DP & Média & DP & \\
\hline Ib & 0.064 & 0.015 & 0.071 & 0.012 & 0.05 \\
\hline Ig & 0.101 & 0.024 & 0.113 & 0.018 & 0.066 \\
\hline Ir & 0.097 & 0.025 & 0.108 & 0.023 & 0.126 \\
\hline Ire & 0.179 & 0.040 & 0.197 & 0.038 & 0.077 \\
\hline Iir & 0.406 & 0.090 & 0.452 & 0.071 & 0.044 \\
\hline EVI & 0.158 & 0.036 & 0.171 & 0.030 & 0.165 \\
\hline EVI8 & 0.506 & 0.102 & 0.549 & 0.077 & 0.091 \\
\hline NDREI & 0.298 & 0.051 & 0.297 & 0.048 & 0.910 \\
\hline NDVI & 0.611 & 0.068 & 0.615 & 0.051 & 0.808 \\
\hline PSRI & 0.116 & 0.038 & 0.113 & 0.029 & 0.713 \\
\hline NDMI & 0.031 & 0.004 & 0.030 & 0.003 & 0.780 \\
\hline NDLI & 0.538 & 0.083 & 0.473 & 0.075 & 0.004 \\
\hline DMCI & -0.198 & 0.018 & -0.186 & 0.017 & 0.018 \\
\hline MRENDVI & 0.381 & 0.058 & 0.391 & 0.037 & 0.473 \\
\hline CAI & 0.004 & 0.004 & 0.006 & 0.006 & 0.310 \\
\hline
\end{tabular}




\begin{tabular}{c|c|c|c|c|c}
\hline BT & 3452.22 & 843.44 & 3534.79 & 626.82 & 0.687 \\
\hline BVT & 1991.81 & 504.47 & 1915.85 & 485.23 & 0.571 \\
\hline BST & 1460.41 & 584.04 & 1618.94 & 377.34 & 0.250 \\
\hline PBV & 58.18 & 10.06 & 53.96 & 8.07 & 0.096 \\
\hline PBS & 41.82 & 10.06 & 46.04 & 8.07 & 0.096 \\
\hline
\end{tabular}

Fonte: Elaborada pelos autores.

*Resultados descritos em média e desvio-padrão (DP). $p$ = nível de significância. Testes t de Student para amostras independentes e Mann-Whitney para a comparação entre as variáveis, considerando significativo $\mathrm{p}<0,05$ (IC 95\%).

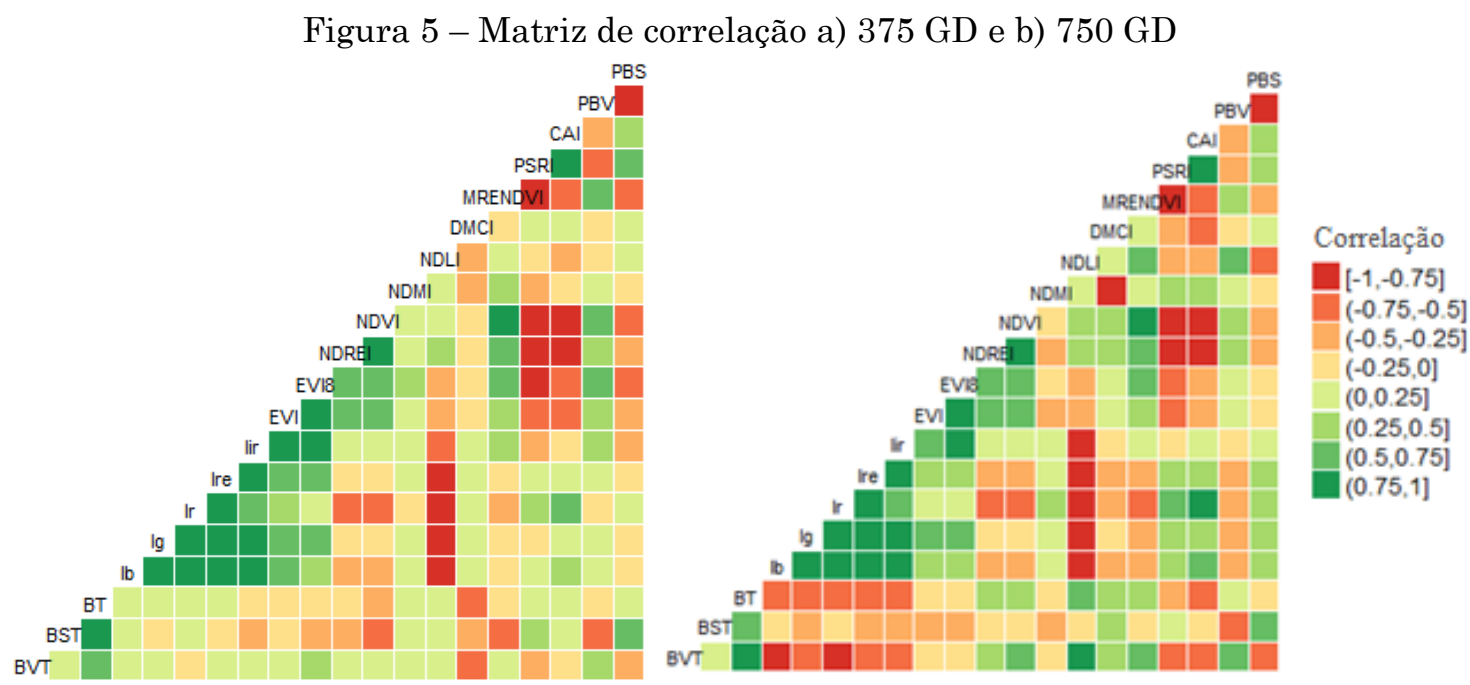

Fonte: Elaborada pelos autores.

Para uma análise mais minuciosa, gerou-se as matrizes de correlação separadamente para cada piquete (Figura 6). Cabe destacar as fortes correlações observadas com os índices (CAI, PSRI, DMCI e NDLI) que abrangem comprimentos de ondas nas faixas onde as respostas de biomassa seca e/ou senescente se acentuam, indicando a presença de biomassa neste estágio. Através das matrizes é possível verificar que os dados no tratamento 750 GR estão melhor correlacionados com a biomassa, isto é justificado com as cores mais intensas (proporcional ou inversamente proporcional) nas matrizes dos 5 piquetes.

Observando a forte correlação existente entre as variáveis obtidas por detecção remota com a biomassa realizou-se regressão multivariada de primeira ordem para gerar equações que possibilitassem estimativas de biomassa. Na Tabela 5 são apresentadas as equações obtidas para cada tratamento seguidas de seus coeficientes. Em função da característica do 
banco de dados e para fortalecer a relação entre os dados, as unidades amostrais de BT, BVT e BST foram transformadas para escala de logaritmo natural para aplicação das regressões.

Figura 6 - Correlação dos dados entre os piquetes no tratamento 375 GD (a) e tratamento
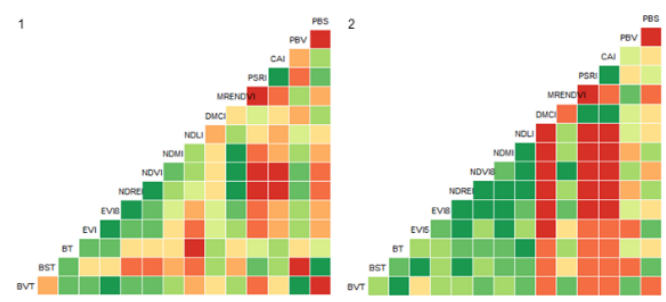
750 GD (b)
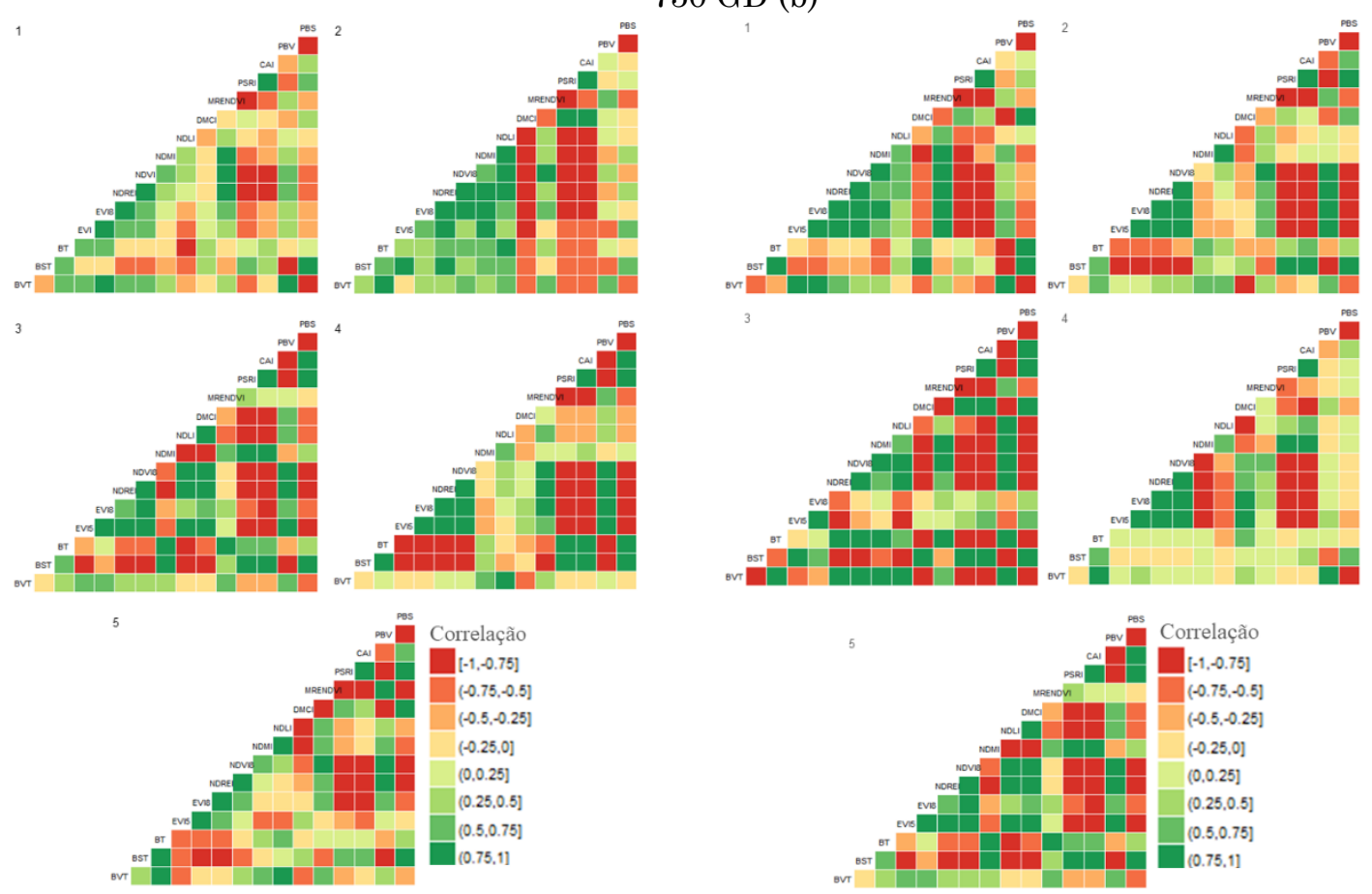

Fonte: Elaborada pelos autores.

Com a inferência da regressão multivariada constata-se que os dados do tratamento 750 GD apresentam maior homogeneidade resultando em modelos com maiores acurácias. $\mathrm{O}$ modelo que melhor se ajustou aos dados considerou os índices CAI e EVI8 como variáveis preditoras da Biomassa Total $\left(R^{2}=0.722\right.$ e RMSE $\left.=0.100\right)$ para dados do tratamento 750 GD. O modelo mais acurado utilizando dados do tratamento 375 GD considerou o EVI, DMCI e NDMI para predizer a BVT $\left(R^{2}=0.46\right.$ e RMSE $\left.=0.190\right) . \mathrm{Na}$ Figura 7 estão exibidas as dispersões dos valores reais vs os valores preditos através das estimativas.

Verificou-se forte relação dos índices CAI, PSRI, NDLI e DMCI com a biomassa, os quais estão presentes diretamente em todas as equações para estimativa de biomassa, exceto na equação para BVT do tratamento 750 . 
Tabela 5 - Regressão multivariada

\begin{tabular}{l|c|c|c|c}
\hline \multicolumn{1}{c}{ Biomassa } & Equação & $\begin{array}{c}\boldsymbol{R}^{2} \\
\text { ajusted }\end{array}$ & RMSE & p-valor* \\
\hline Verde & $\begin{array}{r}B=5.40+\left(1.02^{*} \mathrm{EVI}\right)+\left(-11.01^{*} \mathrm{DMCI}\right)+ \\
\left(-17.41^{*} \mathrm{NDMI}\right)\end{array}$ & 0.460 & 0.190 & 0.880 \\
\hline Senescente & $B=5.25+(-7.30 * \mathrm{DMCI})+(4.39 * \mathrm{PSRI})$ & $<0.400$ & 0.316 & 0.908 \\
\hline Total & $B=7.87+\left(-7.46^{*} \mathrm{DMCI}\right)+\left(-1.88^{*} \mathrm{NDVI}\right)$ & 0.400 & 0.17 & 0.777 \\
\hline & $+\left(-18.06^{*} \mathrm{CAI}\right)$ & & \\
\hline Verde & $B=6.70+\left(1.94^{*} \mathrm{NDLI}\right)+\left(-16.42^{*} \mathrm{CAI}\right)$ & 0.700 & 0.135 & 0.742 \\
\hline Senescente & $\begin{array}{r}\text { Tratamento 750 } \\
\left(-8.23^{*} \mathrm{PSRI}\right)+\left(-4.13^{*} \mathrm{NDREI}\right)\end{array}$ & 0.562 & 0.140 & 0.951 \\
\hline Total & $B=9.15+\left(-1.51^{*} \mathrm{EVI} 8\right)+\left(-26.76^{*} \mathrm{CAI}\right)$ & 0.722 & 0.100 & 0.975 \\
\hline
\end{tabular}

Fonte: Elaborada pelos autores.

*Para equações que seguem normalidade $(p>0.05)$ foi utilizado o t test e para dados não normais $(\mathrm{p}<0.05)$ utilizou-se Mann Whitney test.

Figura 7 - Dispersão valores reais vs estimados com a devida reta de regressão
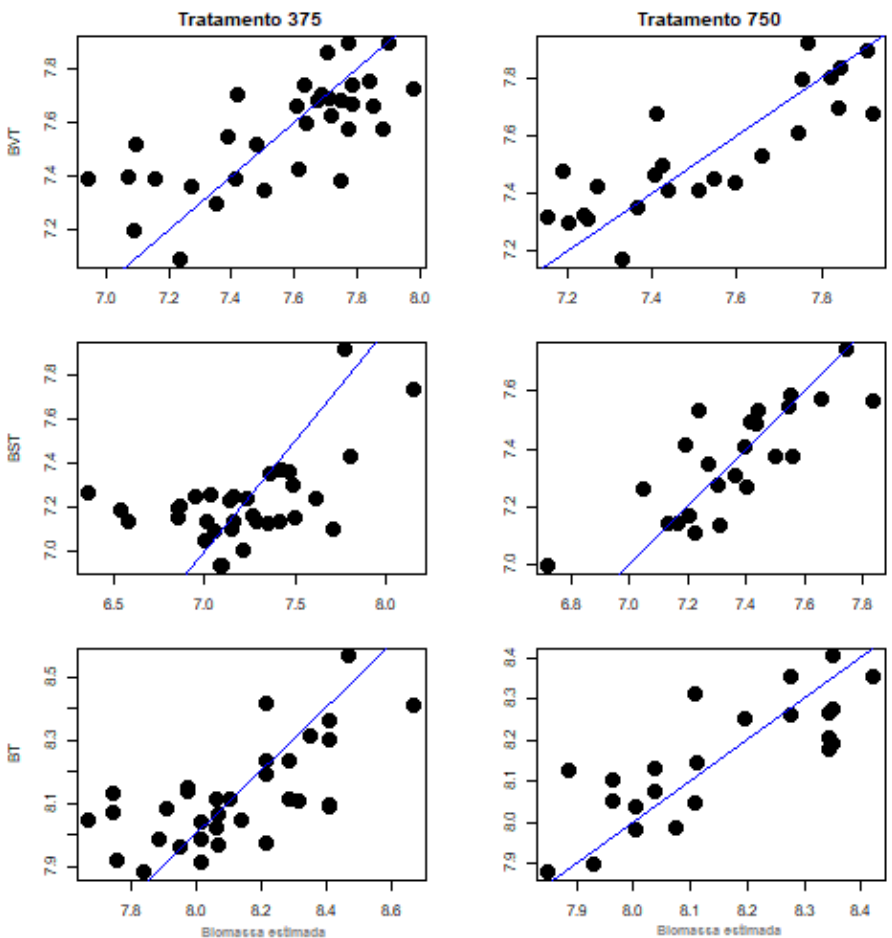

Fonte: Elaborada pelos autores. 


\section{Discussão}

A presente pesquisa avaliou a acurácia de variáveis preditoras hiperespectrais para estimar a biomassa de campos nativos utilizando dados do espectrorradiômetro FieldSpec ${ }^{\circledR} 3$ em dois métodos de manejo pastoril. Devido à presença de altos teores de biomassa senescente (25 - 60\%) característica da vegetação campestre, observou-se a importância de dados nas faixas espectrais (notadamente do infravermelho médio) que possibilitem a interpretação deste componente, uma vez que índices comumente utilizados são mais sensíveis à biomassa verde.

Este estudo avaliou os modelos de regressão estatística em função do coeficiente de determinação ajustado ( $R^{2}$ ajustado $)$ e pelo erro médio quadrático (RMSE). Os resultados se aproximam do estudo realizado por Marabel e Alvarez-Taboada (2013) que avaliaram a biomassa acima do solo utilizando diferentes regiões do espectro e visualizaram modelos com acurácia e erros em torno de 0.80 e $15 \%$, respectivamente.

As pesquisas realizadas por Brantley, Zinnert e Young (2011); Cho e Skidmore (2009); Moreira, Fontana e Kuplich (2015); Trentin et al. (2011) verificaram que, com a utilização de apenas um índice de vegetação, é possível avaliar a dinâmica temporal da vegetação, porém não apresentaram bons resultados para estimar a biomassa. Neste trabalho foi possível verificar que os índices se relacionaram de forma significativa com a biomassa e a combinação de dois ou mais índices geraram modelos mais acurados corroborando com os estudos de Hill (2013).

Considerando os índices para detecção de biomassa senescente, o presente estudo corrobora os achados de Nagler et al. (2003); Serrano, Penuelas e Ustin (2002) e Wang et al. (2011b) constatando que, conforme a biomassa senescente aumenta, os índices CAI, NDLI e DMCI também aumentavam. Já para a biomassa verde, os índices NDVI e EVI tiveram significativas correlações, corroborando com outros autores Barrachina, 
Cristóbal e Tulla (2015); Hmimina et al. (2013); Moreira, Fontana e Kuplich (2015) e Zhou et al. (2014).

Apesar da importância ambiental dos ecossistemas campestres, poucos são os estudos de espectrorradiômetria para conhecimento e entendimento da dinâmica de biomassa campestre. Nota-se que em função de grande variabilidade e biodiversidade presente nas formações campestres, não existe um consenso do melhor conjunto de variáveis para estimativa de biomassa como por exemplo Clevers et al. (2007); Li et al. (2014); Marabel e AlvarezTaboada (2013); Maselli et al. (2013); Ren, Chen e An (2017) e Wang (2011a) apontam diferentes conjuntos de dados para avaliações de biomassa vegetal. Nesta pesquisa, visualizou-se que a combinação de índices sensíveis a diferentes formas e estádios fenológicos da vegetação campestre, apresentase como a melhor opção para analisar e estimar a biomassa vegetal da área de estudo.

\section{Conclusão}

Cada índice estudado apresentou características especificas que devem ser ainda exploradas para ambientes campestres. Percebeu-se que quando as condições da unidade amostral apresentavam menor percentual de matéria senescente os índices EVI, NDVI, MRENDVI foram mais correlacionados com a biomassa. Já os índices CAI, PSRI, NDLI, DMCI apresentaram maior robustez nas amostras com maior percentual de biomassa senescente. A combinação de pares de índices espectrais - um para biomassa senescente e outro para biomassa verde - apresenta-se como boa opção para melhorar a precisão geral da estimativa de biomassa.

A utilização de índices de vegetação apresenta-se como uma excelente opção para a caracterização e estimativa de biomassa de áreas campestres. Nesta abordagem, observou- se que a combinação entre os índices EVI e CAI tiveram a melhor acurácia para a área de estudo com $R^{2}=0.72$ e RMSE $=$ 0.10. A presença de biomassa senescente influencia diretamente as avaliações 
das variáveis espectrais com os dados de biomassa evidenciando a importância da utilização de dados e índices obtidos por sensores hiperespectrais. A utilização destes resultados em escala regional será possível com o lançamento das futuras missões orbitais com sensores hiperespectrais, já em planejamento e testes pela NASA e Alemanha (KAMPE e GOOD, 2017; PALUBINSKAS et al., 2017). Igualmente, alguns dos resultados apresentados aqui podem ser replicados com a utilização dos dados Sentinel 2. Os resultados desta pesquisa são importantes para apurar preditores hiperespectrais para monitoramento e estimativa de biomassa de campos nativos do Bioma Pampa.

\section{Agradecimentos}

Este trabalho foi financiado pela Coordenação de Aperfeiçoamento de Pessoal de Nível Superior - CAPES (processo ${ }^{\circ} 1586394$ ) e apoiado pela Universidade Federal do Rio Grande do Sul (UFRGS), pela Universidade Federal de Santa Maria (UFSM) e pelo Instituto Nacional de Pesquisas Espaciais (INPE), Brasil. Os autores gostariam de agradecer a Jéssica Schuster e Diniz Arruda pelo apoio técnico fornecido para o estudo. Aos funcionários do Laboratório de Ecologia de Pastagens Naturais (LEPAN) da UFSM, agradecemos os dados.

\section{Contribuição dos autores}

Conceitualização, investigação, metodologia, validação, Marildo Guerini Filho e Tatiana Mora Kuplich; Software, análise formal, redação preparação do documento original, Marildo Guerini Filho; redação - revisão e edição, supervisão, Tatiana Mora Kuplich. 


\section{Referências}

BARRACHINA, M., CRISTÓBAL, J., TULLA, A.F. Estimating above-ground biomass on mountain meadows and pastures through remote sensing. International Journal of Applied Earth Observation. 38, 2015. https://doi.org/10.1016/j.jag.2014.12.002

BOLFE, É.L., BATISTELLA, M., FERREIRA, M.C. Correlação de variáveis espectrais e estoque de carbono da biomassa aérea de sistemas agroflorestais. Pesquisa Agropecuária Brasileira 47, 1261-1269. 2012. https://doi.org/10.1590/S0100-204X2012000900011

BRANTLEY, S.T., ZINNERT, J.C., YOUNG, D.R. Application of hyperspectral vegetation indices to detect variations in high leaf area index temperate shrub thicket canopies. Remote Sensing Environmental. 115, 514-523. 2011. https://doi.org/10.1016/j.rse.2010.09.020

CHO, M.A., SKIDMORE, A.K. Hyperspectral predictors for monitoring biomass production in Mediterranean mountain grasslands: Majella National Park, Italy. International Journal Remote Sensing. 30, 499-515. 2009. https://doi.org/10.1080/01431160802392596

CLEVERS, J.G.P.W., VAN DER HEIJDEN, G.W. A. M., VERZAKOV, S., SCHAEPMAN, M.E. Estimating grassland biomass using SVM band shaving of hyperspectral data. Photogrammetric Engineering and Remote Sensing. 73, 1141-1148. 2007. https://doi.org/10.14358/PERS.73.10.1141

CORDEIRO, J.L.P., HASENACK, H. Cobertura vegetal atual do Rio Grande do Sul. In: PILLAR, V. D.; MÜLLER, S. C.; CASTILHOS, Z. M. S.; JACQUES, A. V. A. Campos Sulinos: conservação e uso sustentável da biodiversidade. Ministério do Meio Ambiente, Brasilia - DF, pp. 285-299. 2009.

CRUZ, P., DE QUADROS, F.L.F., THEAU, J.P., FRIZZO, A., JOUANY, C., DURU, M., CARVALHO, P.C.F. Leaf traits as functional descriptors of the intensity of continuous grazing in native grasslands in the South of Brazil. Rangeland Ecology \& Management. 63, 350-358. 2010. https://doi.org/10.2111/08-016.1

GITELSON, A., MERZLYAKB, M.N. Quantitative experiments estimation of chlorophyll-u using reflectance with autumn chestnut and maple leaves and spectra. Journal Photochemistry Photobiology B: Biology. 22, 247-252. 1994. https://doi.org/10.1016/1011-1344(93)06963-4

GONZÁLEZ-DUGO, M.P., MATEOS, L. Spectral vegetation indices for benchmarking water productivity of irrigated cotton and sugarbeet crops. Agricultural Water Management. 95, 48-58. 2008. https://doi.org/10.1016/ j.agwat.2007.09.001

HAYDOCK, K.P., SHAW, N.H. The comparative yield method for estimating dry matter yield of pasture. Aust. J. Agric. Anim. 15, 66-70. 1975.

HILL, M.J. Vegetation index suites as indicators of vegetation state in grassland and savanna: An analysis with simulated SENTINEL 2 data for a North American 
transect. Remote Sensing Environmental. 137, 94-111. 2013. https://doi.org/10.1016/j.rse.2013.06.004

HMIMINA, G., DUFRÊNE, E., PONTAILLER, J.Y., DELPIERRE, N., AUBINET, M., CAQUET, B., DE GRANDCOURT, A., BURBAN, B., FLECHARD, C., GRANIER, A., GROSS, P., HEINESCH, B., LONGDOZ, B., MOUREAUX, C., OURCIVAL, J.M., RAMBAL, S., SAINT ANDRÉ, L., SOUDANI, K. Evaluation of the potential of MODIS satellite data to predict vegetation phenology in different biomes: An investigation using ground-based NDVI measurements. Remote Sensing Environmental. 132, 145-158. 2013. https://doi.org/10.1016/j.rse.2013.01.010

HUETE, A.R., LIU, H.Q., BATCHILY, K., VAN LEEUWEN, W. A Comparison of Vegetation Indices over a Global Set of TM Images for EOS-MODIS. Remote Sensing Environmental. 59, 440-451. 1997.

KAMPE, T.U., GOOD, W.S. Pathway to future sustainable land imaging: the compact hyperspectral prism spectrometer. SPIE 10402. 2017. https://doi.org/10.1117/12.2270932

LI, F., CHEN, W., ZENG, Y., ZHAO, Q., WU, B., Improving estimates of grassland fractional vegetation cover based on a pixel dichotomy model: A case study in Inner Mongolia, China. Remote Sensing. 6, 4705-4722. 2014. https://doi.org/10.3390/rs6064705

MARABEL, M., ALVAREZ-TABOADA, F. Spectroscopic determination of aboveground biomass in grasslands using spectral transformations, support vector machine and partial least squares regression. Sensors (Switzerland). 13, 10027-10051. 2013. https://doi.org/10.3390/s130810027

MASELLI, F., ARGENTI, G., CHIESI, M., ANGELI, L., PAPALE, D. Simulation of grassland productivity by the combination of ground and satellite data. Agric. Ecosystems Environmental. 165, 163-172. 2013. https://doi.org/10.1016/j.agee.2012.11.006

MERZLYAK, M.N., GITELSON, A.A., CHIVKUNOVA, O.B., RAKITIN, V.Y. Nondestructive optical detection of leaf senescence and fruit ripening. Physiologia Plantarum. 106, 135. 1999.

MOREIRA, A., FONTANA, D.C., KUPLICH, T.M. Aplicação de ondaleta em série temporal NDVI/MODIS para avaliação da fenologia de fisionomias campestres no Rio Grande do Sul. Anais do XVII Simpósio Bras. Sensoriamento Remoto (SBSR), João Pessoa-PB, 7, 6381-6388. 2015. https://doi.org/10.1017/CBO9781107415324.004

NAGLER, P.L., INOUE, Y., GLENN, E.P., RUSS, A.L., DAUGHTRY, C.S.T. Cellulose absorption index (CAI) to quantify mixed soil-plant litter scenes. Remote Sensing Environmental. 87, 310-325. 2003. https://doi.org/10.1016/j.rse.2003.06.001

NUMATA, I., ROBERTS, D.A., CHADWICK, O.A., SCHIMEL, J., SAMPAIO, F.R., LEONIDAS, F.C., SOARES, J. V., Characterization of pasture biophysical properties and the impact of grazing intensity using remotely sensed data. 
Remote Sensing Environmental. 109, 314-327. 2007. https://doi.org/10.1016/j.rse.2007.01.013

OVERBECK, G.E., MÜLLER, S.C., FIDELIS, A., PFADENHAUER, J., PILLAR, V.D.P., BLANCO, C.C., BOLDRINI, I.I., BOTH, R., FORNECK, E.D. Os Campos Sulinos: um bioma negligenciado. In: PILLAR, V. D.; MULLER, S. C.; CASTILHOS, Z. M. S.; JACQUES, A.V.A. (Ed.), Campos Sulinos conservação e uso sustentável da biodiversidade. MMA, Brasilia - DF, pp. 28-40. 2009.

PALUBINSKAS, G., BACHMANN, M., CARMONA, E., GERASCH, B., KRAWCZYK, H., MAKARAU, A., SCHNEIDER, M., SCHWIND, P. Image products from a new german hyperspectral mission en map. 2017.

REN, S., CHEN, X., AN, S. Assessing plant senescence reflectance index-retrieved vegetation phenology and its spatiotemporal response to climate change in the Inner Mongolian Grassland. International Journal Biometeorol. 61, 601612. 2017. https://doi.org/10.1007/s00484-016-1236-6

ROMERO, A., AGUADO, I., YEBRA, M. Estimation of dry matter content in leaves using normalized indexes and prospect model inversion. International Journal Remote Sensing. 33, 396-414. 2012. https://doi.org/10.1080/01431161.2010.532819

SERRANO, L., PENUELAS, J., USTIN, S.L. Remote sensing of nitrogen and lignin in Mediterranean vegetation $\backslash r$ rrom AVIRIS data: $\backslash r$ Decomposing biochemical from structural signals. Remote Sensing Environmental. 81, 355-364. 2002.

SIMS, D.A., GAMON, J.A. Relationships between leaf pigment content and spectral reflectance across a wide range of species, leaf structures and developmental stages. Remote Sensing Environmental. 81, 337-354. 2002. https://doi.org/10.1016/S0034-4257(02)00010-X

STRECK, E. V. Solos do Rio Grande do Sul, 2a. ed. Revista e ampl. Porto Alegre: Emater-RS, Porto Alegre. 2008.

TRENTIN, A.B., KUPLICH, T.M., MOREIRA, A., GARAGORY, F., QUADROS, F. Relação da biomassa aérea com a resposta espectral de parcelas de campo com diferentes tipos de manejo. Anais do XV Simpósio Bras. Sensoriamento Remoto (SBSR), Curitiba, PR, Brasil, 2011. https://doi.org/10.1152/jn.00167. 2006

TUCKER, C.J. Red and photographic infrared linear combinations for monitoring vegetation. Remote Sensing Environmenyal. 8, 127-150. 1979. https://doi.org/10.1016/0034-4257(79)90013-0

ULLAH, S., SI, Y., SCHLERF, M., SKIDMORE, A.K., SHAFIQUE, M., IQBAL, I.A. Estimation of grassland biomass and nitrogen using MERIS data. International Journal of Applied Earth Observation. 19, 196-204. 2012. https://doi.org/10.1016/j.jag.2012.05.008 
WANG, L., HUNT, E.R., QU, J.J., HAO, X., DAUGHTRY, C.S.T. Towards estimation of canopy foliar biomass with spectral reflectance measurements. Remote Sens. Environ. 115, 836-840. 2011a. https://doi.org/10.1016/j.rse.2010 .11.011

WANG, L., QU, J.J., HAO, X., HUNT, E.R, Estimating dry matter content from spectral reflectance for green leaves of different species. International Journal Remote Sensing. 32, 7097-7109. 2011b. https://doi.org/10.10 80/01431161.2010.494641

XU, D., GUO, X., LI, Z., YANG, X., YIN, H, Measuring the dead component of mixed grassland with Landsat imagery. Remote Sensing Environmental. 142, 3343. 2014. https://doi.org/10.1016/j.rse.2013.11.017

YANG, X., XU, B., JIN, Y., QIN, Z., MA, H., LI, J., ZHAO, F., CHEN, S., ZHU, X. Remote sensing monitoring of grassland vegetation growth in the BeijingTianjin sandstorm source project area from 2000 to 2010. Ecological Indicators. 51, 244-251. 2015. https://doi.org/10.1016/j. ecolind.2014.04.044

ZHOU, Y., ZHANG, L., XIAO, J., CHEN, S., KATO, T., ZHOU, G. A comparison of satellite-derived vegetation indices for approximating gross primary productivity of grasslands. Rangeland Ecology \& Management. 67, 9-18. 2014. https://doi.org/10. 2111/REM-D-13-00059.1 